\title{
A Dual Launch Robotic and Human Lunar Mission Architecture
}

\author{
David L. Jones ${ }^{1}$ and Jack Mulqueen ${ }^{2}$ \\ Marshall Space Flight Center, Huntsville, Alabama, 35812 \\ Tom Percy ${ }^{3}$ \\ Marshall Space Flight Center, Huntsville, Alabama, 35812 \\ Brand Griffin ${ }^{4}$ \\ Marshall Space Flight Center, Huntsville, Alabama, 35812 \\ and \\ David Smitherman ${ }^{5}$ \\ Marshall Space Flight Center, Huntsville, Alabama, 35812
}

This paper describes a comprehensive lunar exploration architecture developed by Marshall Space Flight Center's Advanced Concepts Office that features a science-based surface exploration strategy and a transportation architecture that uses two launches of a heavy lift launch vehicle to deliver human and robotic mission systems to the moon. The principal advantage of the dual launch lunar mission strategy is the reduced cost and risk resulting from the development of just one launch vehicle system. The dual launch lunar mission architecture may also enhance opportunities for commercial and international partnerships by using expendable launch vehicle services for robotic missions or development of surface exploration elements. Furthermore, this architecture is particularly suited to the integration of robotic and human exploration to maximize science return. For surface operations, an innovative dual-mode rover is presented that is capable of performing robotic science exploration as well as transporting human crew conducting surface exploration. The dual-mode rover can be deployed to the lunar surface to perform precursor science activities, collect samples, scout potential crew landing sites, and meet the crew at a designated landing site. With this approach, the crew is able to evaluate the robotically collected samples to select the best samples for return to Earth to maximize the scientific value. The rovers can continue robotic exploration after the crew leaves the lunar surface.

The transportation system for the dual launch mission architecture uses a lunar-orbitrendezvous strategy. Two heavy lift launch vehicles depart from Earth within a six hour period to transport the lunar lander and crew elements separately to lunar orbit. In lunar orbit, the crew transfer vehicle docks with the lander and the crew boards the lander for descent to the surface. After the surface mission, the crew returns to the orbiting transfer vehicle for the return to the Earth. This paper describes a complete transportation architecture including the analysis of transportation element options and sensitivities including: transportation element mass to surface landed mass; lander propellant options; and mission crew size. Based on this analysis, initial design concepts for the launch vehicle, crew module and lunar lander are presented. The paper also describes how the dual launch

\footnotetext{
${ }^{1}$ Aerospace Engineer, Advanced Concepts Office, MSFC/ED04.

${ }^{2}$ Aerospace Engineer, Advanced Concepts Office, MSFC/ED04.

${ }^{3}$ Aerospace Engineer, Advanced Concepts Office, MSFC/ED04.

${ }^{4}$ Aerospace Engineer, Advanced Concepts Office, MSFC/ED04.

${ }^{5}$ Space Architect, Advanced Concepts Office, MSFC/ED04.
} 
lunar mission architecture would fit into a more general overarching human space exploration philosophy that would allow expanded application of mission transportation elements for missions beyond the Earth-moon realm.

\section{Mission Description}

$\mathrm{T}$ The Dual Launch Lunar Mission was designed with the goal of reducing cost and complexity while maximizing the science and exploration objectives. The mission requires two of the same heavy lift launch vehicles (HLLV). The first HLLV carries the lander and payloads, while the second carries the crew. The two vehicles are launched six hours apart and rendezvous in Low Lunar Orbit (LLO). This requires that the lander and the crew vehicle perform their own Lunar Orbit Insertion (LOI) propulsive maneuvers. Once the vehicles have successfully performed their insertion burns, they will rendezvous and dock. The crew will then transfer from the Orion capsule to the lander and descend to the surface. After the exploration objectives have been met, the crew will ascend from the lunar surface to the crew vehicle in lunar orbit, transfer back to the Orion capsule, and return to Earth.

One of the unique ways the Dual Launch Lunar Architecture maximizes the science and exploration objectives is in its utilization of human and robotic elements to accomplish the mission. Robotic elements can be delivered in unison with the crew, or as a precursor to the human missions. Specifically, the rover could have a dual mode of operation in which the unmanned operations could be tele-operated, and then manually operated as a mobility element by the crew when they arrive. This provides an expanded area of discovery as well as continuous exploration after the crew departs.

\section{Surface Strategy}

The Dual Launch system architecture approach to lunar exploration uses both astronauts and tele-operated rovers to visit the key scientific sites maximizing the science and exploration of the moon. An objective of lunar scientists is to visit sites that are diverse. This obviously implies that the sites are not next to one another, but spread out over the surface. NASA's Lunar Architecture Requirements Preparatory Study provided a list of 10 key sites to the Exploration System Architecture Study (ESAS). These sites along with the robotic Luna and Surveyor and manned Apollo sites are shown in Figure 1.

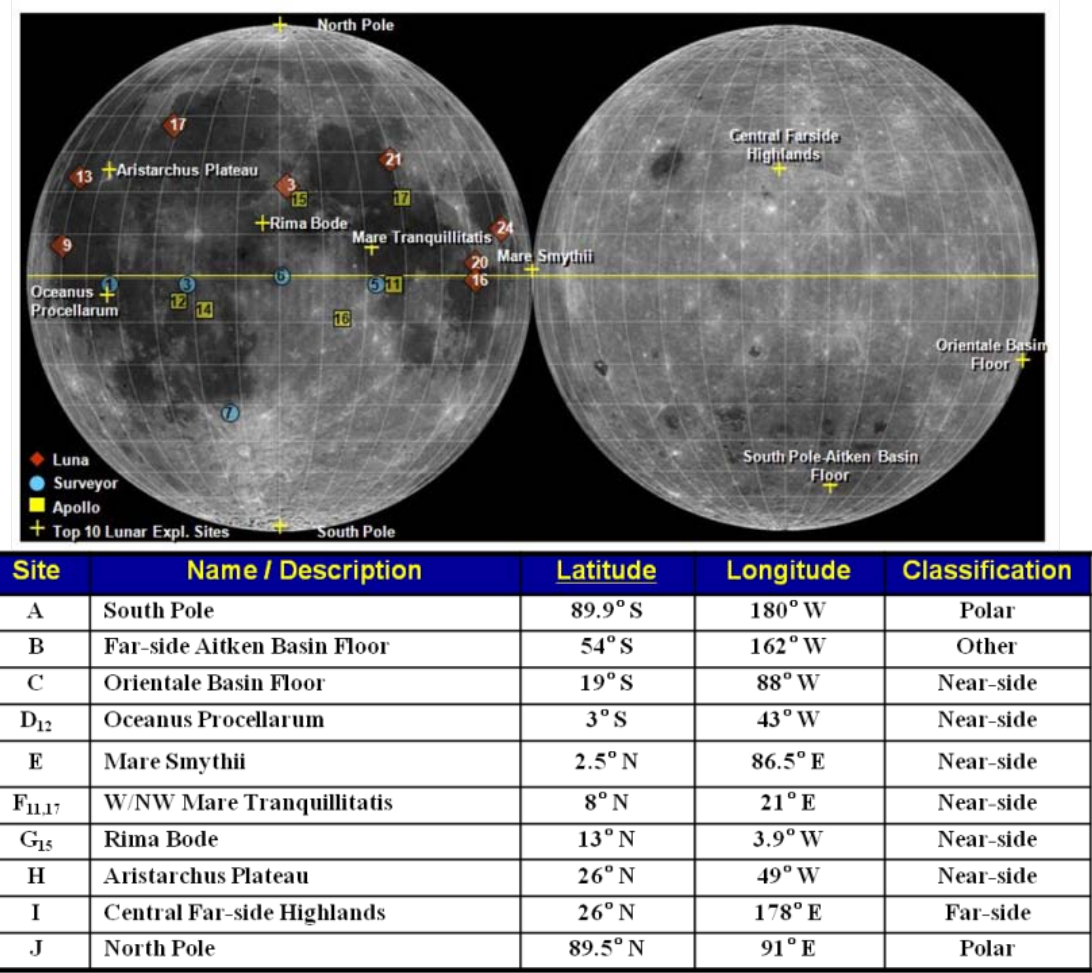

Figure 1. Apollo Exploration Sites. 
Exploration can be classified into two basic modes. One operates out of a fixed base using rovers to explore the key scientific sites. The fixed base mode has the assets to support long stay times, service the rovers and provide ascent for the crew return. This means that the ESAS sites are progressively further away. Excursions from the base to site 1 entail driving to the site, exploring then returning to the base. Site 2 exploration is a longer mission because it still requires exploration but takes more time to get to and from the site.

The other exploration mode has no fixed base but lands at the scientific site then explores the local region. This tends to be a short-stay mission with short-range excursions. The objective of this mode of exploration is to conduct crew activities during one lunar day (14 Earth days)

Apollo 17 geologist astronaut, Jack Schmitt said you could reach the interesting places within close proximity of the lander. This is the "land on science" mode of exploration. The outpost mode requires that the crew travel to the scientific site then travel back to the fixed base to return home. To reach all the ESAS sites, the land on science mode would require 10 landers. Recent Constellation studies of a fixed base include at least 20 flights to bring infrastructure assets, crew and resupplies.

The later Apollo flights had 1 rover, 2 EVA crew and 3 days to explore. Dual mode exploration uses 2 teleoperated or dual mode rovers, 2 EVA crew and up to 12 days to examine the site. The 12 days represents daylight operations allowing 1 day margin on either side. The translucent yellow circles identify the area of daylight exploration or about $300 \mathrm{~km}$ from the ascent vehicle.

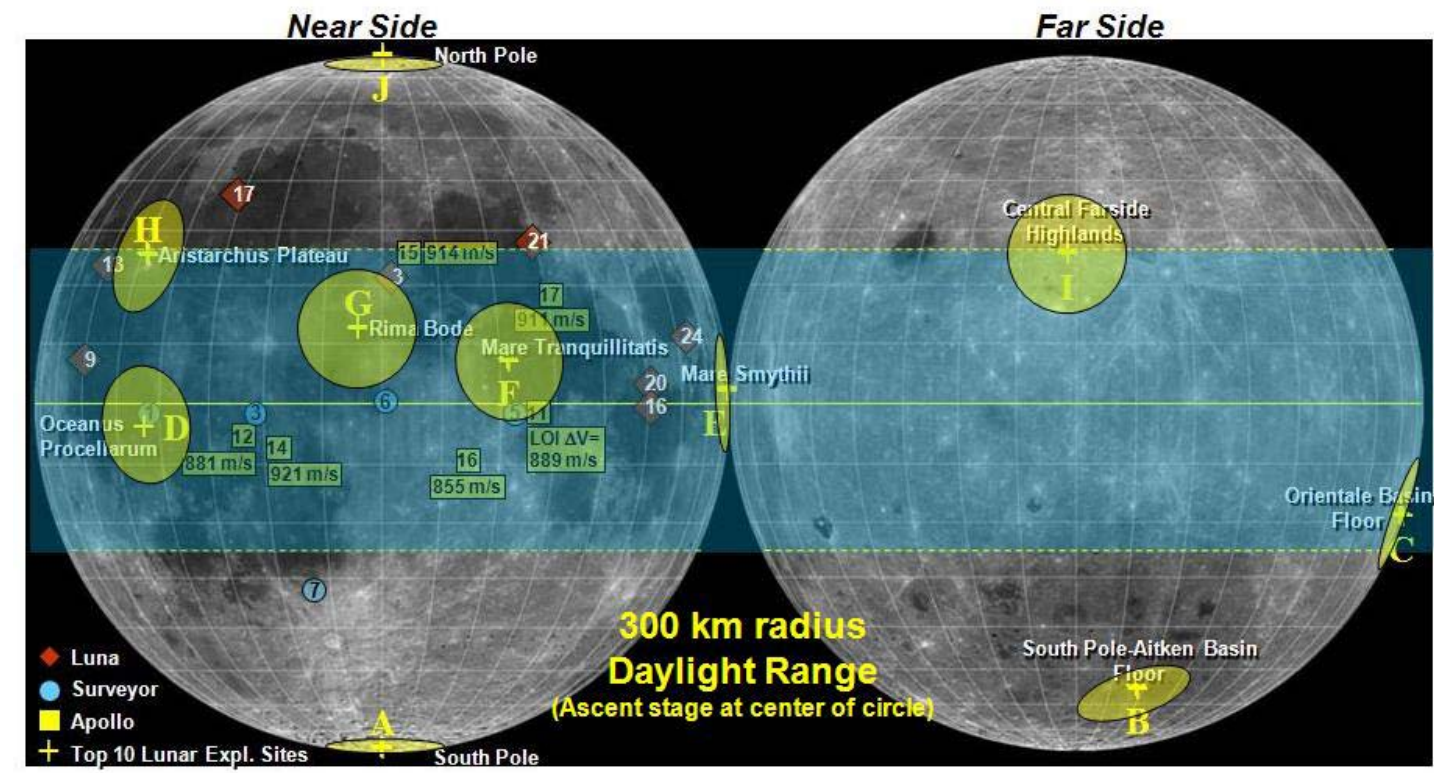

Figure 2. Exploration Range Based on Lunar Daylight Mission.

Dual mode means teleops and crew ops. Rovers tele-operated from Earth analyze and collect samples as well as survey the sites before the crew arrives. With pre-mission robotic reconnaissance, missions can be shorter because they are carefully planned to focus on remaining exploration tasks uniquely suited to human capabilities. To reduce mission risk and mass operations for both the rovers and crew are planned only for lunar daylight.

Exploring the 10 lunar sites allows scientists and engineers to characterize the attributes of each area including the rover path between sites. From this, equipment can be designed and missions planned to take advantage of the local resources. In-situ resource utilization (ISRU) decisions are then made on the site assessment and survey to determine properties and abundance of resources.

Either small pressurized or unpressured rovers are used for continuous surface exploration. Unpressurized rovers worked well for Apollo astronauts providing both speed and range. Pressurized rovers on the other hand, offer radiation protection from Solar Proton Events (SPEs). Because these can come at any time and have only a 20 to 30 warning, the crew should have ready access to a storm shelter. The shelter can add mass so a one-time mass penalty on a surface rover is a better solution than equipping each lander with a shelter. To support the exploration program rovers can be a mix of pressurized and unpressurized delivered by US or international partners. Furthermore, exploration benefits from more, rather than fewer rovers, so they can be distributed at several locations on the Moon. 
There are many solutions to lunar rover design. Shown in Figure 3 is one possible configuration that meets the robotic requirements as well as crew size of the Constellation. In its tele-operational mode, the rover uses sensors and a manipulator to analyze and collect samples. When the crew lands, the rover is there and can accommodate 2 or 4 crew. Then, when the crew leaves, the rover continues to explore.
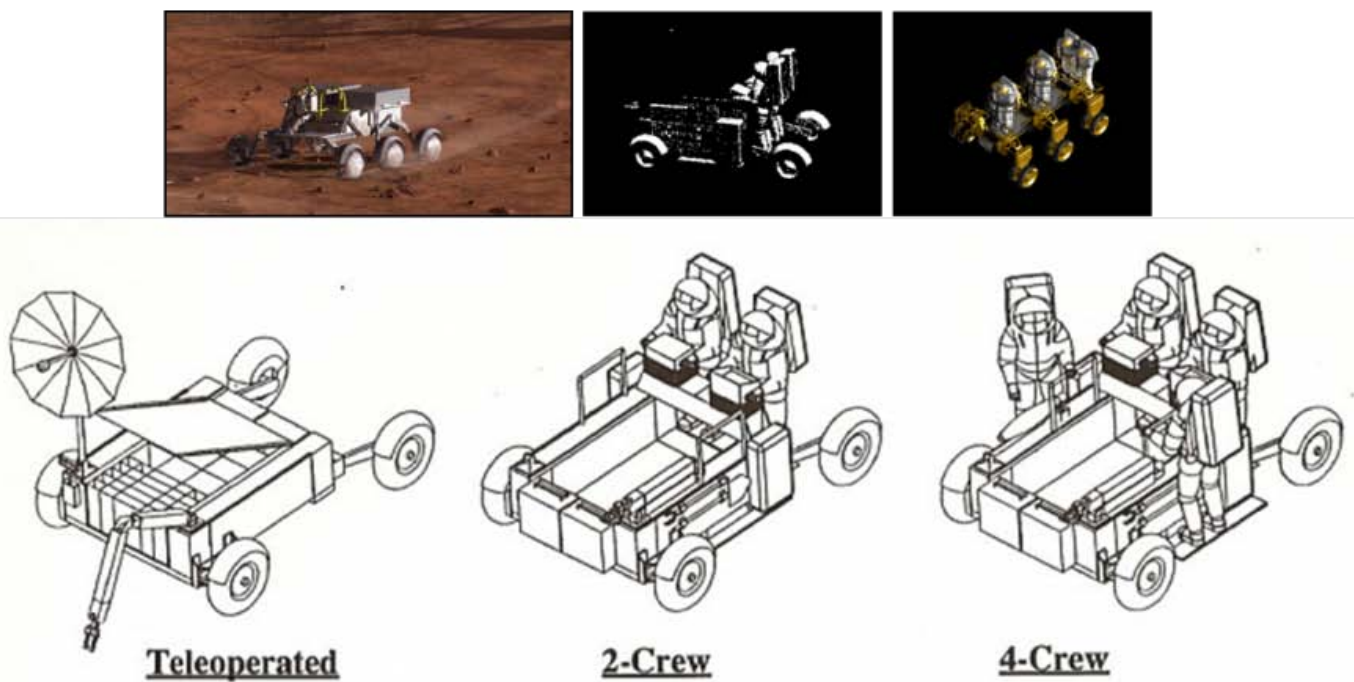

- Photovoltaic/battery
- Daylight ops. only
- Shown with sample bins

$$
\begin{aligned}
& \text { - Rechargeable } \\
& \text { batteries } \\
& \text { - Control bridge } \\
& \text { attached } \\
& \text { - EVA tools } \\
& \text { - Additional PLSS }
\end{aligned}
$$
- Running boards with grab bars
- Intended for short- medium distance with frequent off-on
- Alternative seated position possible

\section{Figure 3. Dual Mode Rover Concept.}

Thorough exploration of the ESAS sites appears possible based on the work accomplished by Apollo 17 astronauts coupled with the pre-mission reconnaissance of tele-operated rovers. Over the course of 3 EVAs during 3 days, the Apollo crew collected $110 \mathrm{~kg}$ of samples, set up 10 science experiments and drove over $30 \mathrm{~km}$. Looking at the range per stay-time for the three Apollo rovers it is possible to cover over a $110 \mathrm{~km}$ in 12 days. This number can vary based on driving straight to a destination or explore-as-you-go.

The mission strategy is laid out in Figure 4. The blue regions represent the general landing area. On the left, the rover is delivered first and sets out to explore. Traveling at the Lunakhod rate, during daylight only at $8 \mathrm{hr} / \mathrm{day}$ for 12 days, the tele-operated rover can travel $2304 \mathrm{~km}$ over a 2 year period (or 2/3 the diameter of the Moon). Traveling at $0.33 \mathrm{~km} / \mathrm{hr}$ it can make $768 \mathrm{~km}$. Along the way 2 sites for crew visit are identified. The rover with samples, then positions itself as a beacon for the crew landing. After landing, the crew sorts through the samples then travels to the 2 sites of interest, conducts the mission and returns to the lander for ascent. The rovers continue to explore after the crew departs. 


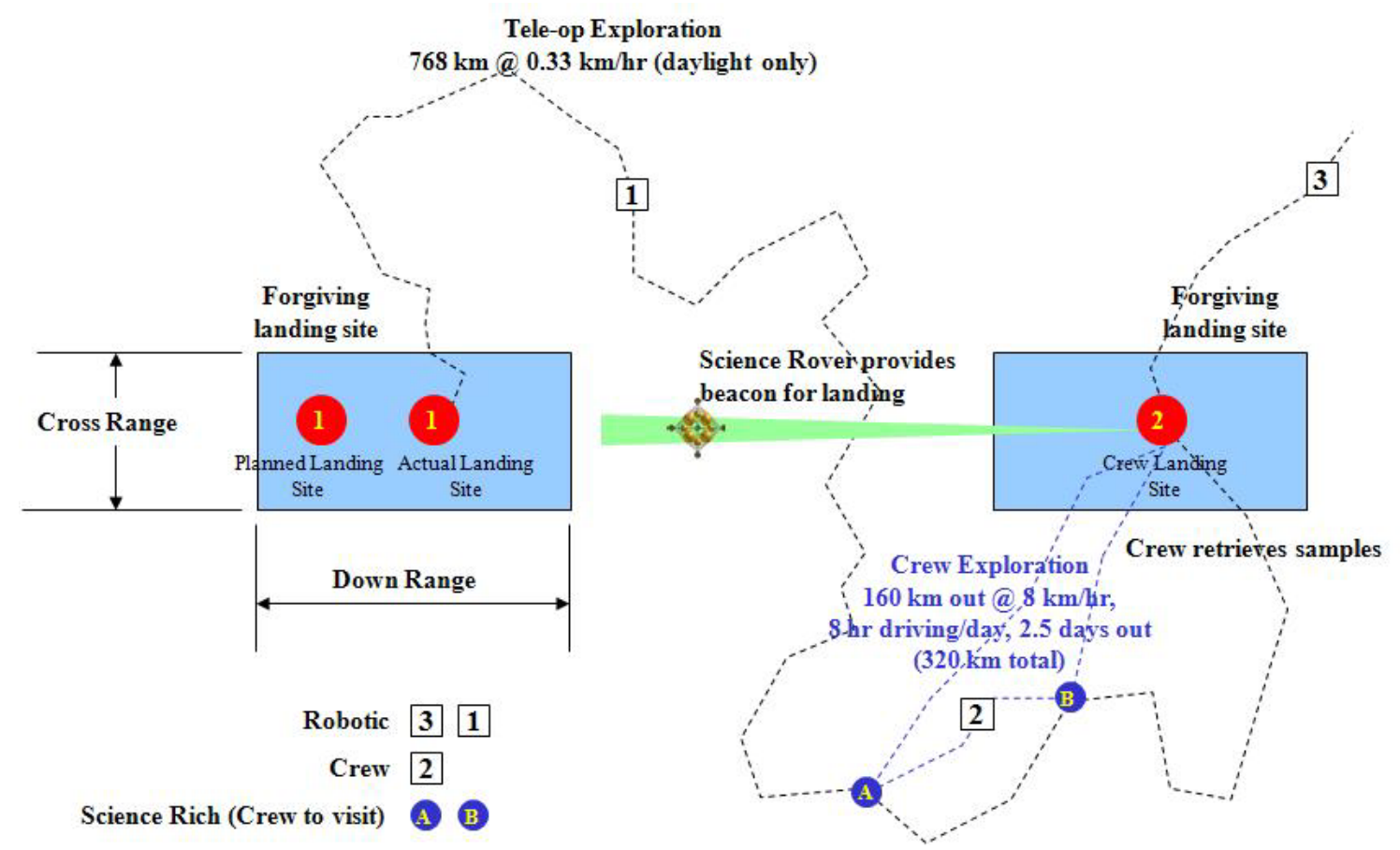

Figure 4. Dual Mode Surface Exploration Strategy.

Short stay times are an advantage to the crew. It is safer because there is reduced radiation risk and less physiological degradation. It is less massive because there are fewer consumables, less energy storage and only requires campout provisions for the crew. Furthermore, because the rover excursions are local, the risks for equipment failure happening a long way from the ascent vehicle are reduced.

\section{Architecture Analysis}

In this architecture study the goal was to define and explore the dual launch lunar architecture trade space. For the past 4 years, most lunar architecture analysis has been centered around the 1.5 launch approach. This included one launch of an HLLV (Ares V) and one launch of a crew vehicle (Ares I). With the increasing lift requirements associated with the HLLV launch in the 1.5 launch architecture approaches, it has been suggested that by providing lift to orbit on two equally sized launch vehicles (Ares V Lite) a more effective use of launch infrastructure and a more reasonable HLLV design can be realized.

This study intended to define the trade space of architecture options within the dual launch realm. Within this dual launch architecture trade space, sub-trades were performed on the propellant options for various transportation elements and a performance sensitivity was performed against the mass delivered to the lunar surface.

As in the 1.5 launch study, some new elements were introduced into the architecture trade space. Two launch vehicle optimizations approaches were investigated. The first was the traditional HLLV optimized to TLI using a core stage with rocket boosters and an Earth Departure Stage (EDS) that performed climb-to-orbit and TLI burns. The second was the HLLV optimized to LEO with a dedicated in-space transfer stage known as the Lunar Transfer Stage (LTS). These vehicles have the same basic core and solid approach with a second stage to perform climb-toorbit functions. This allows for a fully loaded LTS to provide the TLI propulsion.

A Lunar Orbit Insertion Stage (LOIS) was also introduced in some options to allow for the inclusion of a descent-only lunar lander. The standard CxP Orion Service Module (SM) was also used. However, because the dual launch architecture by definition does not constrain the size of Orion to fit on an Ares I launch vehicle, the service module was allowed to take on some additional maneuvers in some architecture trade options.

Ground rules for this analysis were established based on expert opinion, previous analysis and existing Constellation baseline data. All payload mass assumptions were based on Constellation baseline data. These assumed values ensured consistency with analyses. Margins were established to combine industry best practices with the current Constellation guidelines for mass margin tracking. The Project Manager's Reserve and Mass Growth Allowance values follow the Constellation guidelines and are consistent with the Altair and Orion analysis approaches. Although the total reserve value is a combination of calculated values that will vary from analysis to 
analysis based on the performance data available propellant reserves are approximately $3.5 \%$. These reserve calculations were based on industry best practices.

One unique aspect of the dual launch lunar orbit rendezvous architecture approach is that there are now two stacks of elements traveling to the moon. Between each stack, propulsion elements must be common to reduce the overall development and production costs. Therefore, unless the work load of each stack is properly balanced there will be propellant offload requirements on one of the two stacks. In order to construct a trade tree that reflected the commonality of elements and the various combinations of work distributions available in a two stack configuration, the architecture operations prior to lunar orbit insertion are assumed to be common. From the LOI burn through the end of the mission, the trade trees are divided into the LOI and descent burn options for the lander stack and the LOI and TEI burn options for the crew stack.

Again, a division of the trade space into families based on the separation point of the launch vehicle is possible. In this trade space, three families are defined. In the first family, the launch vehicle provides the EDS which performs climb-to-orbit, TLI and, in some cases, LOI burns. In the second family, the launch vehicle uses its second stage to provide all climb-to-orbit but a dedicated LTS provides TLI and LOI functionality. In the third family, the LTS also performs a small portion of the climb-to-orbit but significantly less than the EDS in Family A.

Because of limited time and resources, the HLLV options optimized to LEO were not included in this analysis. Instead, the focus was placed on Family A in the trade space. This family has seven architecture options that combine various elements to perform the LOI burns for the lander and crew stacks.

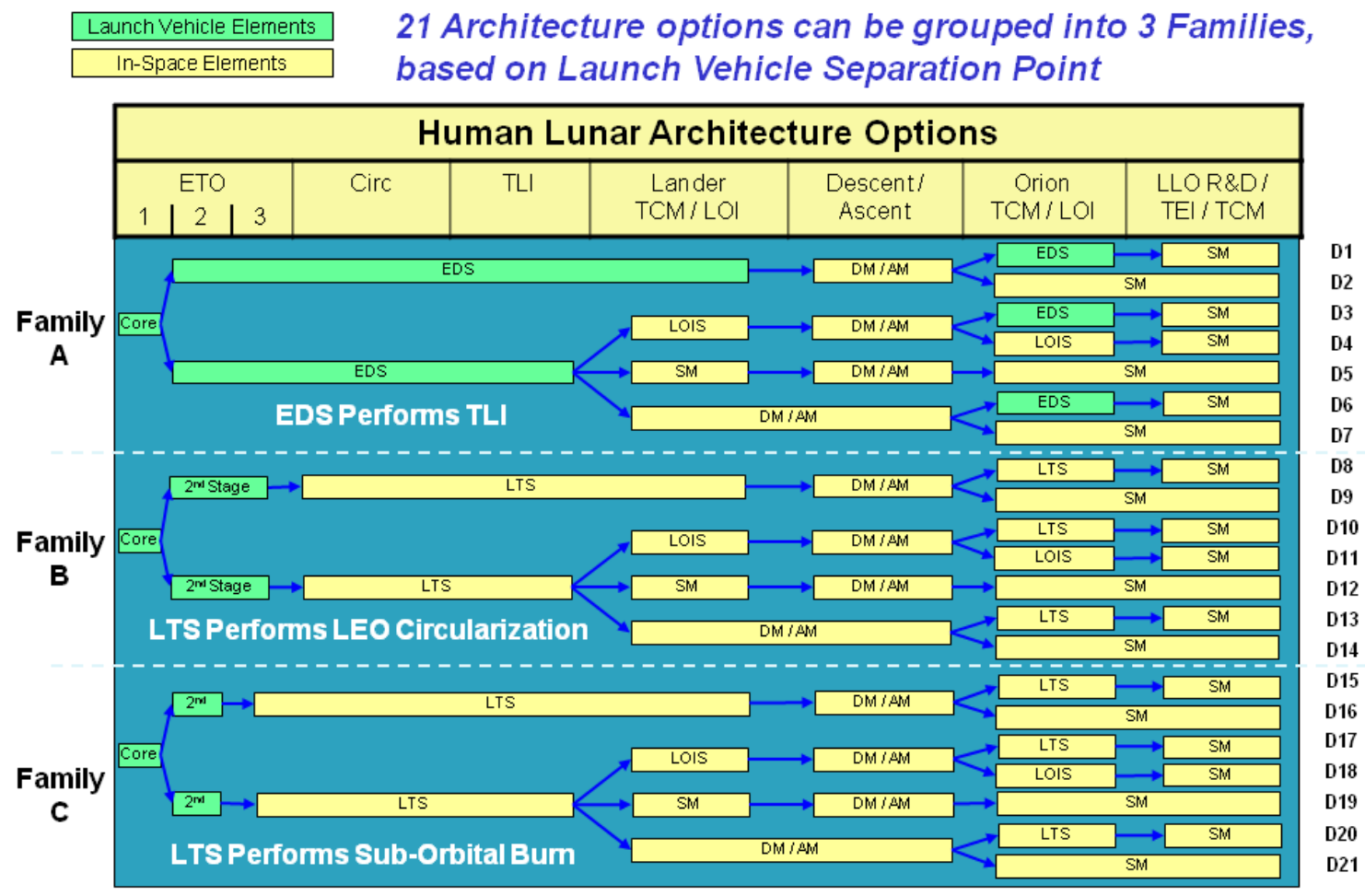

Figure 5. Dual Launch Architecture Families.

For each architecture option the lifted mass to LEO requirement is shown. Due to element commonality ground rules, the launch vehicle must be sized to lift the larger of the two stacks and would, therefore, be offloaded to provide lift for the smaller of the two stacks. In order to maximize the use of the HLLV designed for this architecture approach, it would be desirable for the two stacks to be close in lift requirement. As can be seen in Figure 5, all of the assessed architecture options are dominated by the lift requirement of the crew stack with the exception of architecture option D5.

In Figure 6 the HLLV lift capability is represented by the red dashed line. This capability corresponds to the current Ares V Lite launch vehicle design which has a lift capacity to LEO of approximately $144 \mathrm{mT}$. In order for an architecture option to be considered "closed" from a performance standpoint, both of the stack masses must fall below the red line. 


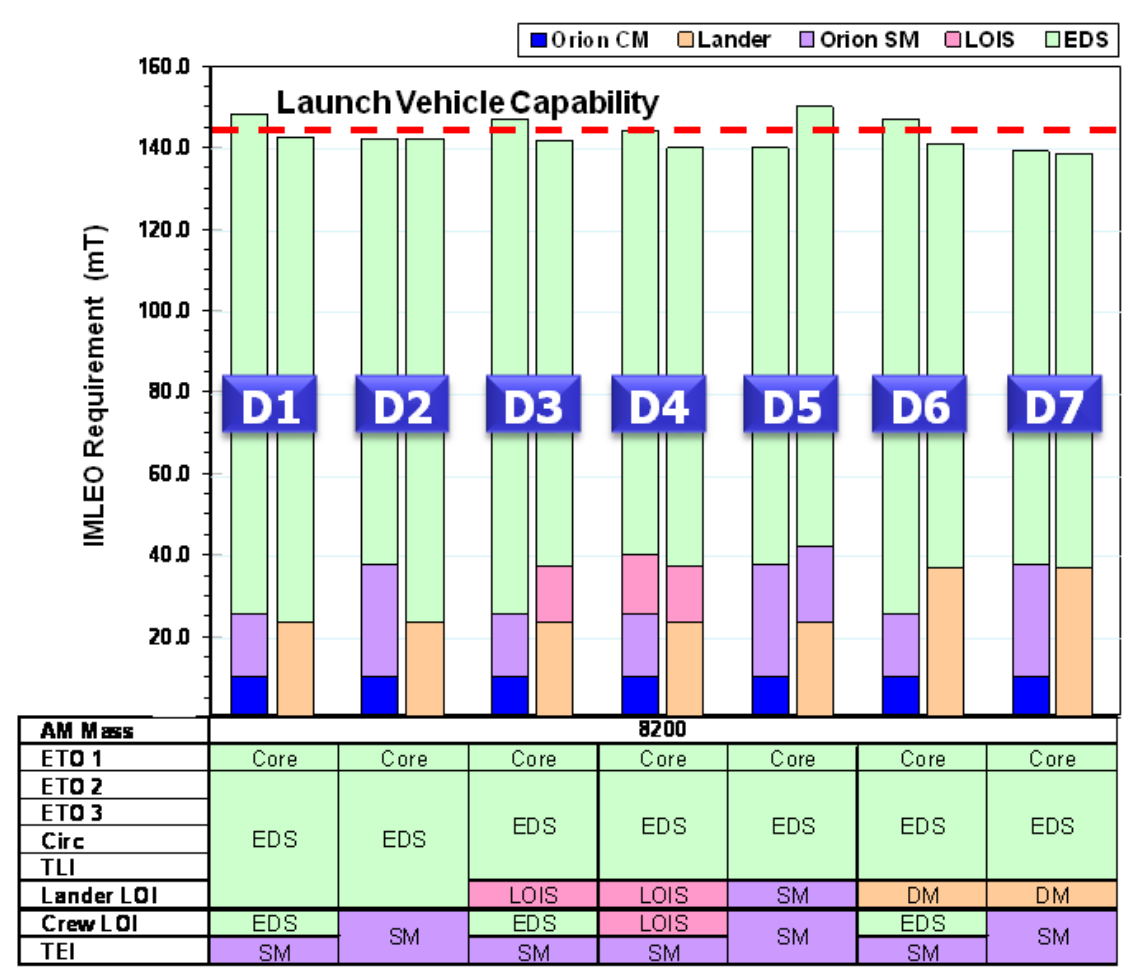

Figure 6. Family A Architecture Performance Summary.

Given the current launch vehicle constraints, only three architecture options have the ability to close. Architecture options D1, D3 and D6 exceed the lift capability with the crew stack. Architecture option D5 exceeds the lift capability with the lander stack. As a result, only architecture options D2, D4 and D7 are carried forward for further review. Both architectures D2 and D7 provide some margin in the launch vehicle, displayed above as a gap between the top of the stack and the red line.

In architecture D2, the EDS is required to perform a large sub-orbital burn, the TLI burn and the LOI burn. Of these three burns, the LOI burn is the least energy intensive and, therefore requires very little propellant when compared to the two previous burns. This presents a design feasibility problem in that, after 4 days in transit to the moon, it will be nearly impossible to provide the necessary settling burn to locate and properly feed the final $10 \%$ of the propellant load to the engines from the large propellant tanks. For this reason, architecture option D2 was also eliminated from consideration.

Architecture option D4 requires a new LOI stage in addition to the other elements of the architecture. This LOI stage provides little to no benefit in lift mass requirement and would add development cost to the architecture. Given that there is another architecture available that can be closed and that does not require an additional stage, architecture option D4 was eliminated on the grounds of higher development cost.

There are several advantages to architecture option D7. First, of the seven options identified, it closes with the current launch vehicle with the most launch margin. Second, the lift requirement for the crew and cargo missions are very close, providing a very high launch vehicle usage efficiency between the two stacks. Third, this option makes the best use of the current architectural elements therefore requiring no more development cost than the current baseline architecture option.

In this architecture, the EDS performs TLI for both stacks. In the case of the lander stack, the lander performs LOI and waits in Lunar orbit for the crew to arrive and rendezvous before ultimately continuing to the lunar surface. The lander is less efficient overall because performs LOI (refer to the findings of the 1.5 Launch study to see the impacts of using partially empty tanks for landing). However, because the lander is not stopping the CEV in lunar orbit, this inefficiency is reduced when compared to the baseline architecture approach. For the crew stack, the Orion service module is used to perform both the LOI and TEI burns. This will require a redesign of the current 
Orion SM to increase the propellant load capacity but in the dual launch scenario, there is no Ares I lift limit to restrict the size of the Orion spacecraft so this larger service module should not present an issue.

The next task in the analysis was to investigate the sensitivity of the selected architecture to propellant choice and mass delivered to the lunar surface. In a departure from the current baseline approach to Lunar architecture assessment, this lander was sized for the crew mission and the cargo payload capacity was allowed to "fall out" of the analysis. Because this lander is being used in a lunar orbit rendezvous dual launch mode, the payload capacity for crew and cargo missions is equal. By parametrically investigating the sensitivity to mass delivered to the lunar surface, we can assess both the crew and cargo mission capabilities of the lander. In later slides we will discuss what delivered mass is appropriate for what size crew and lunar surface duration.

\section{Sensitivity of ETO Requirement to Mass Delivered to Lunar Surface and Lander Propellant Type for Architecture D7}

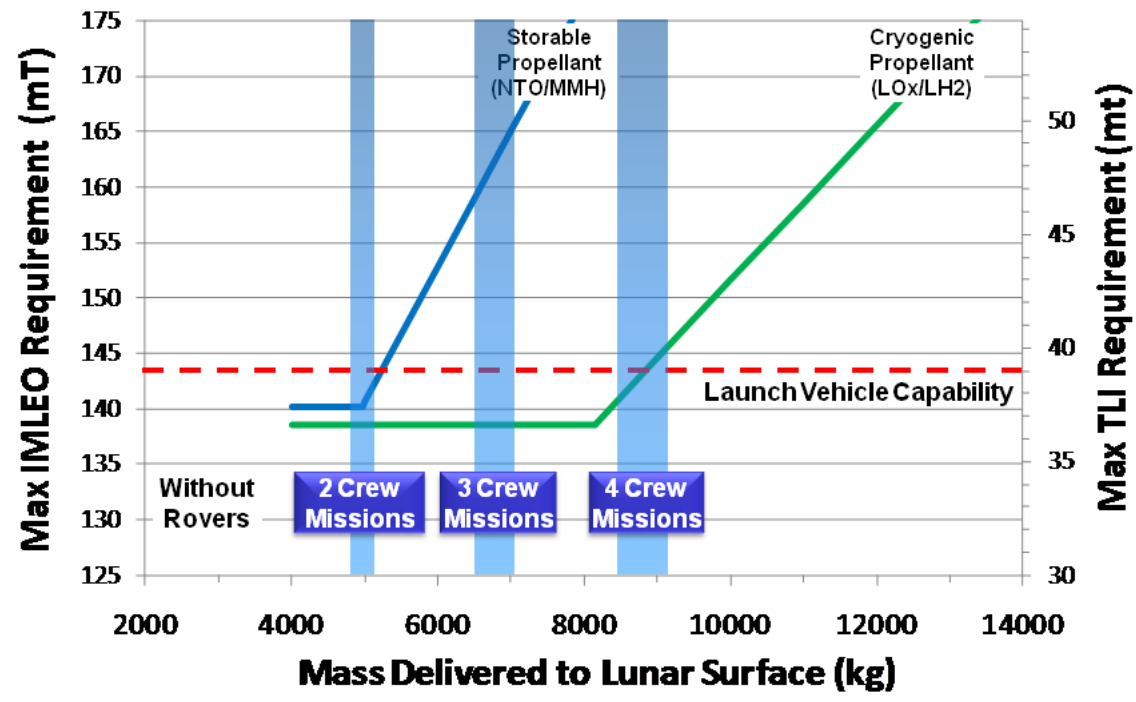

Figure 7. Architecture D7 Performance Sensitivities.

The "hockey stick" chart above (Figure 7) shows the maximum IMLEO requirement for architecture D7 as a function of mass delivered to the lunar surface. There are two lines plotted here, one for a storable propellant (NTO/MMH) lander and one for a cryogenic propellant (Lox/LH2) lander. This approach to the analysis bounds the investigation where a Lox/CH4-based lander would have a line somewhere in between these two.

The shape of the curve results from the relationship between the crew stack and the lander stack. When the surface payload is low, the crew stack will dominate the lift requirement. Because the lift requirement for the crew stack is unaffected by the surface payload, the resulting maximum IMLEO value will be constant as long as the crew stack dominates, thus resulting in a flat line.

As the lunar surface payload value is increased, the size of the lander (and therefore the lift requirement for the lander stack) will increase. As long as the lander stack lift requirement is below the crew stack lift requirement, the sensitivity remains in the flat part of the curve as previously discussed. At one value for the lunar surface payload number, a point is reached where the size of the crew stack and the size of the lander stack are equal. This "point of equality" results in the knee in the curve above. This point represents the point in the sensitivity where the usage of a single, common HLLV is maximized for both launches in the architecture and the launch portion of the mission is at its most efficient.

For both propellant options, this "point of equality" falls below the current lift capability of the Ares V Lite. Because of its higher performance, the cryogenic lander had a higher lunar surface payload value at its "point of equality" and also results in a slightly lower lift requirement. While the storable lander point of equality is slightly above $5 \mathrm{mT}$, the cryogenic lander's point of equality is slightly above $8 \mathrm{mT}$.

For lunar surface payload values above the point of equality, the lander stack dominates the lift requirement. This results in the sloped portion of the curve where the steady increase in surface payload results in a steady increase in lift requirement. When coupled with the launch vehicle capability constraint, the surface payload value for which drives the lift requirement above the lift capability can quickly be identified. 
Due to the lower performance of the storable propellant combinations, the sloped portion of the storable lander line has a slightly steeper slope. This indicates that, as the payload requirement increases, the size of the lander, and therefore the lift requirement for the lander stack, will increase at a slightly higher rate than the more efficient cryogenic lander.

Once the general sensitivity of lift requirement to lunar surface payload for architecture D7 was identified, the next task was overlay the lunar surface delivery requirement for actually human lunar missions and determine where on the curve these requirements fall. The three blue bars overlaid on the plot above represent the lunar surface payload mass required for missions of varying crew size and mission duration. This payload mass includes habitats, crew equipment and consumable required to sustain a crew of a given size for a given amount of time.

From left to right, the three blue bars represent a crew of 2, 3 and 4 crew members. The width of the lines represent how the payload mass requirement is effected by surface mission duration. The left side of the blue bar corresponds to the surface payload requirement for a minimum duration mission of 3 days. The right side of the blue bar corresponds to the surface payload requirement for a maximum duration mission of 12 days. Given those sensitivities, looking at where the maximum IMLEO lines are through the blue bars shows how the lift requirement will vary over those two surface mission parameters (crew size and mission duration).

There are several initial findings depicted in Figure 7. First, a lunar surface mission of any duration with 2 crew members can be closed for both the storable and cryogenic lander. Second, increasing the crew size beyond 2 crew members drives the storable lander well beyond the current lift capacity. Missions with a crew size of 3 fall on the flat part of the cryogenic lander curve at any duration. Missions with a crew size of 4 fall on the sloped part of the cryogenic lander curve but do close as long as the mission duration is kept below approximately 8 days.

Missions with a crew size of 3 still fall on the flat part of the cryogenic lander curve at any duration. Missions with a crew size of 4 fall higher on the sloped part of the cryogenic lander curve than before, meaning that a crew of 4 cannot be delivered with a rover regardless of the mission duration of propellant selected for the lander.

In summary, the storable lander may be used for missions with 2 crew members with only a slightly higher lift requirement than the comparable cryogenic lander. The additional benefits of less development risk, reliability and logistics advantages and the elimination of cryo-fluid management requirements make a storable lander an appealing alternative for these small missions.

For missions with 3 crew members, the storable lander options will only be feasible if more refined lander design optimization results in a reduction in lander mass or the launch vehicle lift capability can be increased. The cryogenic lander option is still dominated by the crew stack lift requirement for the delivery masses required for 3 crew missions which means that all 3 crew missions could be closed by using a cryogenic lander design.

For missions with 4 crew members, the storable lander is not a viable alternative. The cryogenic lander option is feasible for some 4 crew missions but exceeds the current HLLV lift capacity for the longer duration missions. Therefore, a 4 crew mission is possible with the cryogenic lander option but the duration of the mission will be driven by the lift capacity of the launch vehicle.

\section{Transportation System Elements}

The space transportation elements of the Dual Launch Lunar Mission are based on current Constellation approaches, with differences that suit the dual launch philosophy. The heavy lift launch vehicle is a two stage to trans-lunar injection, liquid oxygen/liquid hydrogen system with solid rocket boosters: much like the Ares V launch vehicle. The architecture requires another propulsion system to insert into lunar orbit. The architecture analysis described later in this paper showed that the lander is better suited to perform that maneuver. Also with the difference in philosophy, the Orion service module (SM) was required to perform additional maneuvers resulting in a redesign of the SM. These foundational elements become the infrastructure needed to perform additional missions other than the moon.

As part of the end-to-end architecture assessment, conceptual design and analysis was performed on each of the major transportation elements in order to validate the parametric architecture analysis. Below is a summary of the design of those elements.

\section{A. Launch Vehicle Description}

The heavy lift launch vehicle used for the dual launch mission is an Ares V based liquid oxygen/liquid hydrogen system with solid rocket boosters. The over-arching goal of the launch vehicle analysis was to provide a launch vehicle that was as conservative in the technology assumptions as possible. Main engine characteristics and structural material properties are examples of areas where assumptions were based on heritage values. The core stage propulsion system was assumed to be five RS-68B engines with a specific impulse of 409 seconds and ablative 
nozzles. The solid rocket boosters were assumed to be the ARES I developed five segment boosters. All structural materials were assumed to be the aluminum-lithium construction used in the Shuttle's lightweight external tank. These assumptions provided a launch vehicle with a capability of delivering approximately 43,000 kilograms of payload through the trans-lunar injection (TLI) burn. An additional 10\% payload margin was applied which reduced the payload to approximately 38,700 kilograms. This payload number became the goal to which the space transportation elements designed to.

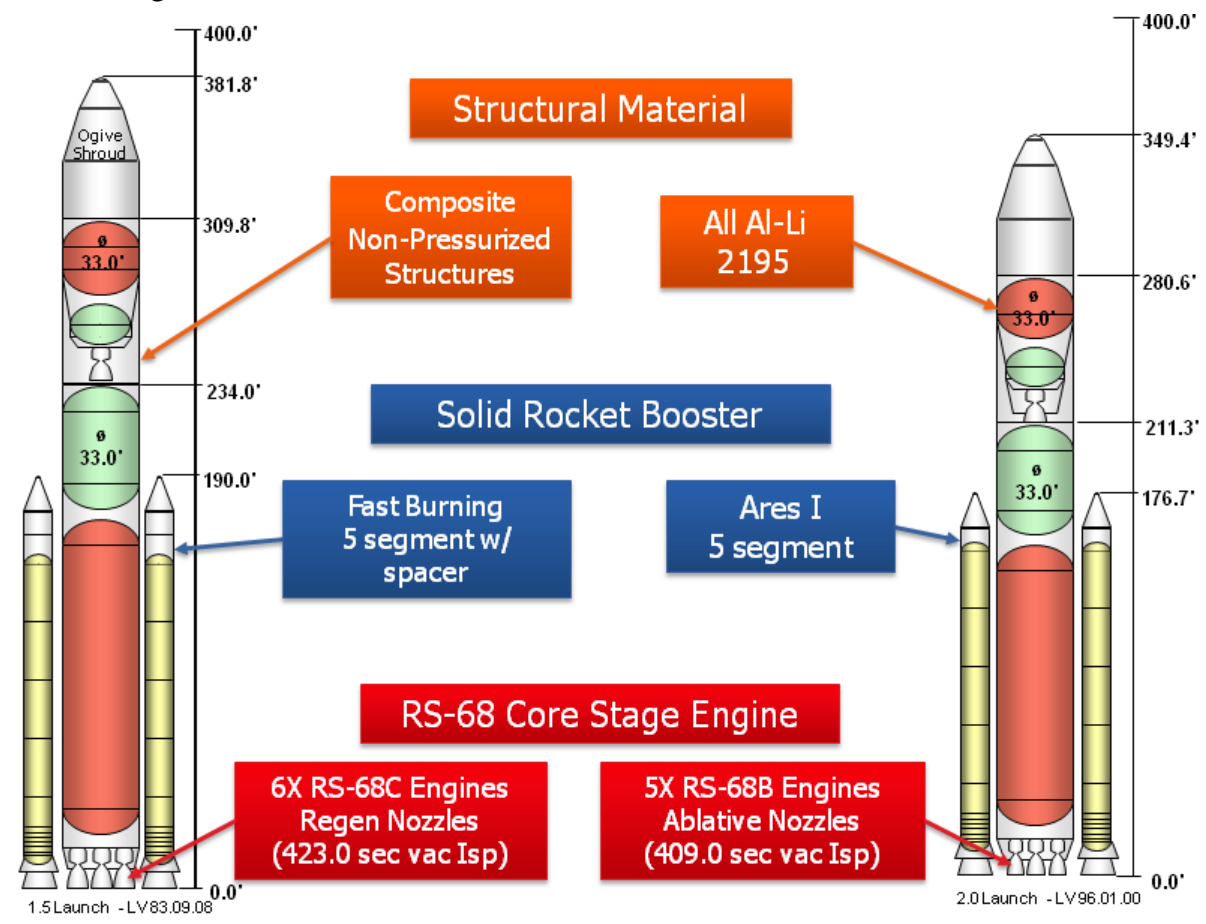

Figure 8. Conceptual Launch Vehicle Comparison.

\section{B. Service Module Concept}

Since the Dual Launch mission architecture calls for a Lunar Orbit Rendezvous of the space transportation elements, the Orion Service Module had to be expanded to incorporate the additional propulsive requirements. The requirement of the SM performing its own LOI burn required an additional propellant load of XXX kilograms. A redesign was performed on the SM itself while leaving the Crew Module as is. The largest impacts were to the propulsion and structures subsystems. Because of the additional propellant, the SM was redesigned to look more like the Apollo era SM. Other subsystems were modified as necessary to accommodate the design changes, but were only slightly affected. 


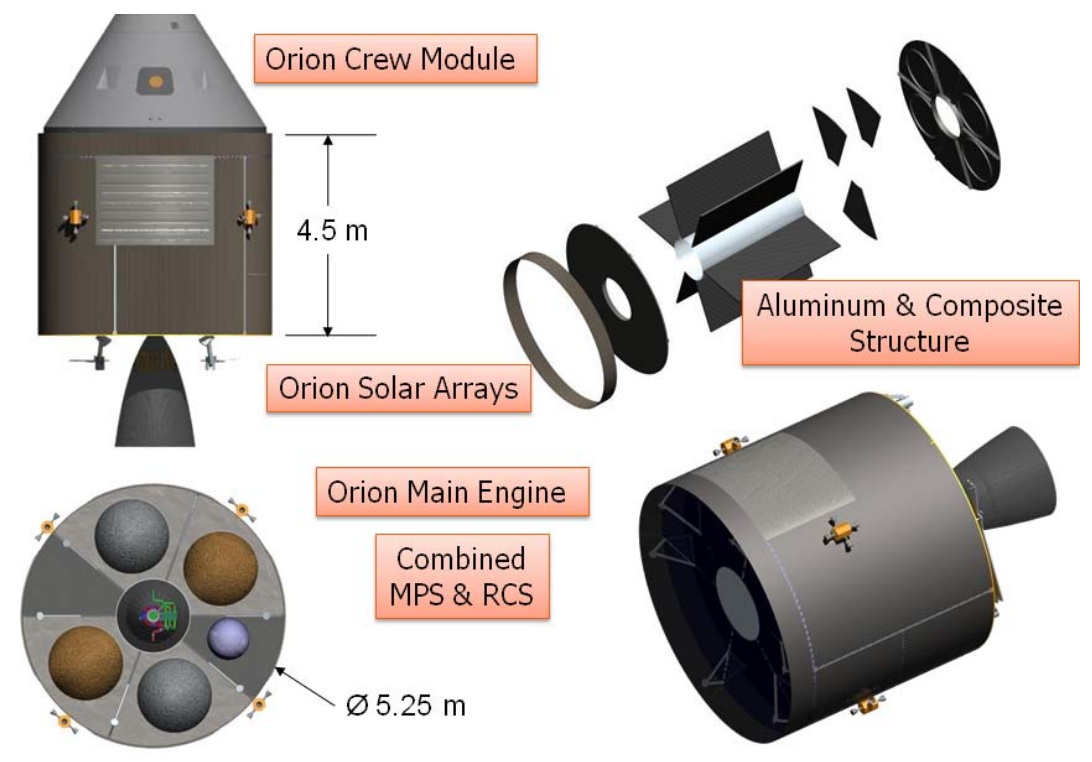

Figure 9. Expanded Service Module Overview.

\section{Lander Design Concept}

The lander design was based on the approach of the Altair lander. However, some changes were made due to the nature of the mission. Since the architecture analysis provided sensitivities to propellant changes, a conceptual design was performed for a LOX/LH2 lander as well as a Storable propellant lander (MMH/NTO). Figure 10 shows a representation of the storable propellant design.

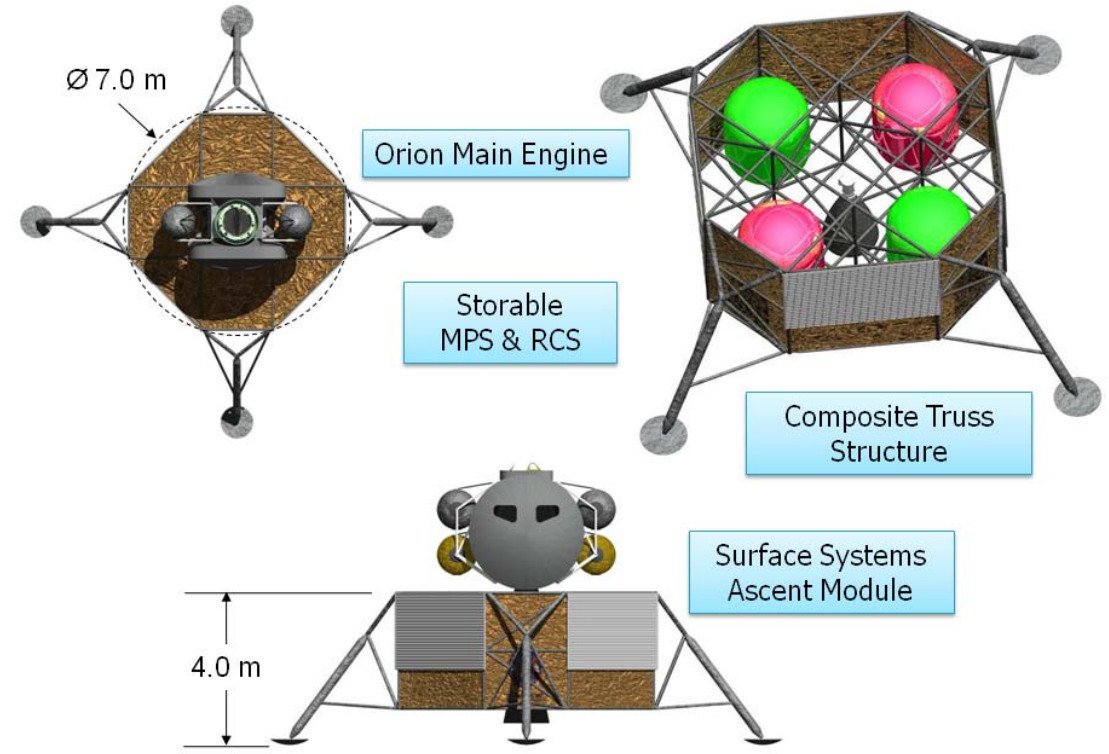

Figure 10. Storable Lander Design Overview.

The storable lander offers many advantages over the cryogenic system, however, there is a significant reduction in payload to the surface. Basic subsystem design philosophies were kept as similar to Altair as possible. Since the lander does not have to carry the Orion CSM through LOI, the propellant required for the lander is reduced as compared to Altair. 


\section{Applications to Expanded Human Exploration}

The dual launch lunar mission architecture would fit into a more general overarching human space exploration philosophy which would allow expanded applications of mission transportation elements for missions beyond the Earth-moon realm. The components of the dual launch architecture become part of a space transportation infrastructure needed to explore other areas of interest. One example of this approach is the Service Module concept. With its expanded capabilities, the Service Module used in the Dual Launch Lunar Mission also contains the necessary propulsive requirements for deep space maneuvering for a low energy Near Earth Object (NEO). Additional crew support systems would need to be included, but the remaining subsystems are robust enough to accomplish the NEO mission. Other transportation elements like the lander and earth departure stage could also be utilized to accomplish other missions as well, including Mars.

\section{Conclusions}

The Dual Launch Lunar Mission was designed with the goal of reducing cost and complexity while maximizing the science and exploration objectives. The Dual Mode surface exploration strategy enables commercial and/or international collaboration for exploration elements. The expanded area of discovery as well as continuous exploration of the lunar surface provides a collaborative option to maximize the scientific goals of lunar exploration.

The comprehensive study intended to define the trade space of architecture options within the dual launch realm. Within this dual launch architecture trade space, sub-trades were performed on the propellant options for various transportation elements and a performance sensitivity was performed against the mass delivered to the lunar surface. In summary, the performance sensitivity shows that for a storable landing system crew size would have to be reduced to two crew. For missions with 4 crew members, the storable lander is not a viable alternative. The cryogenic lander option is feasible for some 4 crew missions but exceeds the current HLLV lift capacity for the longer duration missions. Further optimizations on the launch vehicle and other transportation elements could be performed to add additional crew members or surface duration.

The space transportation elements of the Dual Launch Lunar Mission are based on current Constellation approaches, with differences that suit the dual launch philosophy. These foundational elements become part of an in-space transportation infrastructure needed to perform additional missions other than the moon. 